\title{
Imparting Protection against UV Radiations Using In Situ Coating of Titanium Dioxide on Textiles
}

\author{
Anu Mishra ${ }^{1, *}$, Himansu Shekhar Mohapatra ${ }^{1}$ and B.S. Butola ${ }^{2}$ \\ ${ }^{1}$ Indian Institute of Carpet Technology, Chauri Road, Bhadohi-221401, India \\ 2Indian Institute of Technology, Delhi, Hauzkhas, Newdelhi-110016, India \\ *mishraanu79@gmail.com
}

Keywords: $\mathrm{TiO}_{2}$, UPF, Curing, Hydrothermal, Solvothermal.

\begin{abstract}
Protection against ultraviolet radiation is one of the areas, in which considerable attention is being paid. Application of a wash durable finish on cotton fabric to protect the wearer from these radiations remains a challenge. The harmful effects of UV radiations result in the form of eye disorders, accelerated ageing of skin, DNA damage and cause of cancer. The current study focuses upon the use of $\mathrm{TiO}_{2}$ on cotton fabric to impart UV protection. $\mathrm{TiO}_{2}$ is deposited on cotton fabric using its pre-cursor. Sol-gel method has been used to convert pre-cursor into titanium oxide directly on fabric surface. The deposition was further stabilized on fabric surface by various heat treatments such as curing, hydrothermal and solvothermal treatments. It has been found that the $\mathrm{TiO}_{2}$ remains wash durable with a reasonably high degree of UV protection after hydrothermal and solvothermal treatments. EDX and ICP-MS techniques were used to examine the add-on $\%$ of $\mathrm{TiO}_{2}$ on cotton fabrics. The morphology of the $\mathrm{TiO}_{2}$ coated cotton fabric was studied using Field emission scanning electron microscopy. The measurement of UV protection property of the fabric has been carried out using UV-visible spectrophotometer (UV-2000F, Labsphere, USA).
\end{abstract}

\section{Introduction}

Textiles with enhanced functionalities have been greatly appreciated by the demanding consumer market [1]. Many chemical finishes based on organic origin are being used to impart functionalities like antimicrobial [2], flame retardancy [3], water repellency [4], UV protection [5] etc. into textiles. However, these finishes suffer very poor wash durability. Most of the times, poor fixing of these finishes result in huge environmental pollution in the subsequent washes of textiles.

Application of inorganic materials opens up a new opportunity to develop such functionalities in textiles. These inorganic materials are generally non-toxic and chemically stable under exposure to both high temperature and adverse weather conditions.

Textiles have been considered as good substrates to give protection against harmful ultraviolet radiations [6]. The extent, to which a fabric transmits, absorbs or reflects UV radiation determines its UV protection properties. The transmission, absorption and reflection depend mainly on the fiber/fabric constructional parameters and the finishing treatment applied [7]. Metal oxides like $\mathrm{TiO}_{2}$ and $\mathrm{ZnO}$ are being used as good UV blockers in suns creams to protect the skin from harmful UV radiations.

The UV radiations are harmful to human bodies and some of their harmful effects include DNA damage, accelerated ageing of skin and eye disorders. With respect to human health and environment, UV radiations have been classified as black light UVA (380-315 nm), medium wave UVB $(315-280 \mathrm{~nm})$ and germicidal UVC $(280 \mathrm{~nm}-100 \mathrm{~nm})$. Among these three, UVC radiation is extremely dangerous to human health [8].

The deposition of $\mathrm{TiO}_{2}$ nanoparticles on textile substrates has many challenges. There are requirements of good compatibility between textile substrate and the chemicals used during deposition of these nanoparticles on the surface. Wash durability is also one of the major concern upon which very few researchers have paid serious attention. Several studies have reported that $\mathrm{TiO}_{2}$ coating on textiles could be performed using different pretreatments and techniques [9-11]. 
Some of the pretreatments done on textile substrate to develop affinity with $\mathrm{TiO}_{2}$ include RFplasma, MW-plasma, and UV-irradiation etc [12]. The application of UV absorbing materials is done using different techniques such as spraying, padding, deposition, and knife-coating. One way to graft nano $\mathrm{TiO}_{2}$ on cotton is by the use of cross-link agent as reported by Chen and his co-workers [13]. In a study carried out by Vigneswaran and his team, nano-sol of $\mathrm{TiO}_{2}$ was synthesized in water as well as ethanol medium using sol-gel technique. It was applied on cotton fabric using acrylic binder through pad-dry-cure [14]. Sule and his team fabricated a multilayer film of $\mathrm{TiO}_{2}$ on modified cotton fabric through layer-by-layer molecular self-assembly technique [15].

The available studies suggest that most of the researchers have neglected the ratio of precursor to fabric weight and hence did not focus on the ultimate add-on of $\mathrm{TiO}_{2}$ on textile fabric. In addition to this, the role of the process type (pad-dry-cure, pad-dry-hydrothermal and pad-dry-solvothermal) on the development of $\mathrm{TiO}_{2}$ morphology on fabric and its effect on UV protection property (UPF) has not been studied.

Hence in this paper, an attempt has been made to correlate the process type, the pre-cursor fabric weight ratio and treatment time on the UV protection property of the fabric. Systematic studies on effect of process route on behavior of $\mathrm{TiO}_{2}$ deposition on fabric and eventually on the UV protection properties of the fabric have also been discussed.

\section{Materials and Methods}

\subsection{Materials}

Cotton fabric with ends per inch $=124$, picks per inch $=124 / 64$ and gsm=150 was supplied by Vardhman fabrics, Budhani. Titanium tetra iso-propoxide (TTIP) was purchased from Spectrochem, Mumbai. In sol-gel preparation, analytical grade glacial acetic acid and ethyl alcohol were used. Deionized water was used for preparation of sol, whenever required.

\subsection{Preparation of $\mathrm{TiO}_{2}$ sol}

Titanium Tetra Isopropoxide (TTIP) was used as a precursor for the synthesis of $\mathrm{TiO}_{2}$ sol. Preparation of 1\% v/v TTIP sol was done in 50:50 ethanol /DI water solvent system. One mL of pure TTIP was stirred with $10 \mathrm{~mL}$ of pure ethanol for $10 \mathrm{~min}$. One $\mathrm{mL}$ of glacial acetic acid was stirred with $10 \mathrm{~mL}$ of pure ethanol separately for 5 min and then added in the $1^{\text {st }}$ mixture drop wise. $30 \mathrm{~mL}$ of pure ethanol was mixed separately with $50 \mathrm{~mL}$ DI water and this aqueous ethanol was added drop wise to acidified TTIP/ethanol solution with continuous stirring. Finally, the prepared sol was stirred for another $4 \mathrm{~h}$.

\subsection{Application on cotton fabric}

$15 \times 15 \mathrm{~cm}^{2}$ (approx. $3 \mathrm{~g} \mathrm{wt}$ ) samples of untreated cotton fabric (UNT) were taken. Each sample was dipped in a prepared sol of TTIP, followed by sonication for a period of $10 \mathrm{~min}$. Further, each sonicated sample was padded at a pressure of 2.75 bar and dried at $80^{\circ} \mathrm{C}$. The heat treatment to each fabric sample was subsequently given by curing (sample A), hydrothermal (sample B) or solvothermal method (sample C).

Curing was conducted at $120^{\circ} \mathrm{C}$ in hot air for a period of $3 \mathrm{~min}$. Hydrothermal treatment was given by transferring the sample in IR dyeing cylinder containing $100 \mathrm{~mL}$ of DI water. It was treated for a period of $3 \mathrm{~h}$ at $120^{\circ} \mathrm{C}$. Solvothermal treatment was given by transferring the sample in IR dyeing cylinder containing $100 \mathrm{~mL}$ of $\mathrm{TiO}_{2}$ sol. It was also treated for a period of $3 \mathrm{~h}$ at $120^{\circ} \mathrm{C}$.

\subsection{Characterization}

The deposition of $\mathrm{TiO}_{2}$ particles on cotton fabric surface was studied using field emission scanning electron microscope (FESEM), model FEI Quanta 200 F SEM. Energy dispersive X-ray (EDX) analysis was carried out to obtain the elemental composition.

High-resolution inductively coupled plasma mass spectrometry (ICP-MS, make: Agilent 7900) was used for the determination of total amount of $\mathrm{Ti}$ in treated fabric samples. A given amount of 
sample was digested in a microwave-assisted acidic digester at $300^{\circ} \mathrm{C}$ for $28 \mathrm{~min}$. Spectral interferences arising from the plasma gases or the major components of the samples were identified and removed. Finally, the amount of $\mathrm{Ti}$ in the textile sample was determined on microgram per gram (ppm) basis [16].

ISO 105 C10: 2006 - B was adopted to evaluate the performance of the treated samples after standard wash. The treated samples are washed in laundro-meter at $50^{\circ} \mathrm{C}$ temperature using $5 \mathrm{gpl}$ of soap. The material to liquor ratio was kept as 50:1 and the washing cycle was completed in 45 min.

The UV protection property of the fabric was measured using UV-visible spectrophotometer (UV-2000F, Labsphere, USA). The UV-2000F measures the diffuse transmittance of textile samples in the wavelength region from $250-450 \mathrm{~nm}$. Using this instrument, UV Protection Factor (UPF) of the fabric is recorded. On the basis of recorded data, UV protection rating of the fabric is calculated as per AATCC 183:2010 standards.

\section{Results and Discussion}

\subsection{SEM of $\mathrm{TiO}_{2}$ coated cotton samples}

The SEM studies of $\mathrm{TiO}_{2}$ coated cotton fabrics are shown in Fig. 1. The untreated cotton fabric (UNT) does not show any deposition on its surface. But, it can easily be observed that in case of sample preparation through PDC method, there is formation of thin layer of $\mathrm{TiO}_{2}$ on fabric surface. In PDH method, the texture of $\mathrm{TiO}_{2}$ turns granular, whereas in case of PDS higher concentration of these granules can be seen. Therefore, it can be inferred that the texture of $\mathrm{TiO}_{2}$ deposited on cotton surface can be altered depending upon the method used for application of heat treatment in the samples.
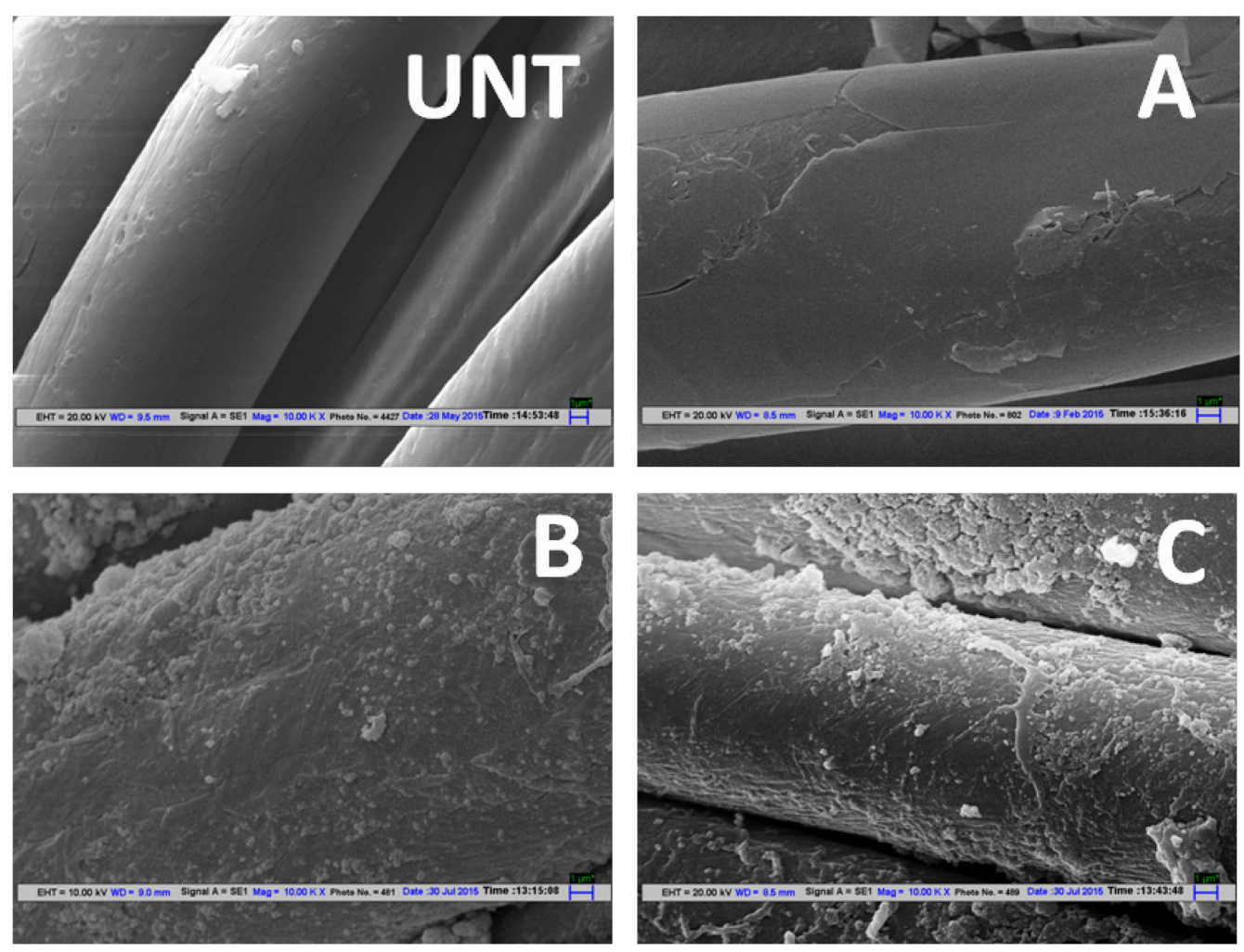

Figure 1. SEM of $\mathrm{TiO}_{2}$ coated cotton fabrics prepared by various methods (UNT) Untreated, (A) PDC, (B) PDH and (C) PDS 


\subsection{Measurement of Titania content in fabric}

The content of Ti on cotton fabric is measured using ICP-MS and the results are shown in Table 1. Measurement of Ti content through ICP-MS technique suggests that the add-on level of Ti is similar in case of PDC (sample A) and PDH (sample B) and is higher in case of sample prepared by PDS method (sample C). Moreover, comparing the Ti content of samples after 5 washes with their $1^{\text {st }}$ standard wash samples, it can be seen from that wash fastness of B and C are better than that of A, which suggests that hydrothermal or solvothermal process provides a more durable deposition of $\mathrm{TiO}_{2}$ than a pad-dry-cure process.

Table 1. Ti content in cotton fabrics (using ICP-MS technique) prepared by different methods (after $1^{\text {st }}$ and $5^{\text {th }}$ wash)

\begin{tabular}{|c|c|c|c|c|}
\hline Sample code & Process & $\begin{array}{c}\text { Ti content in } \mathbf{~ p p m} \\
\left(\mathbf{a f t e r} \mathbf{1}^{\text {st }} \mathbf{w a s h}\right)\end{array}$ & $\begin{array}{c}\text { Ti content in } \mathbf{~ p p m} \\
\left(\mathbf{a f t e r}^{\text {th }} \mathbf{w a s h}\right)\end{array}$ & $\begin{array}{c}\text { \% reduction in } \\
\text { Ti content }\end{array}$ \\
\hline UNT & -- & -- & -- & -- \\
\hline A & PDC & 530 & 388 & $26.8 \%$ \\
\hline B & PDH & 536 & 512 & $4.5 \%$ \\
\hline C & PDS & 748 & 710 & $5.1 \%$ \\
\hline
\end{tabular}

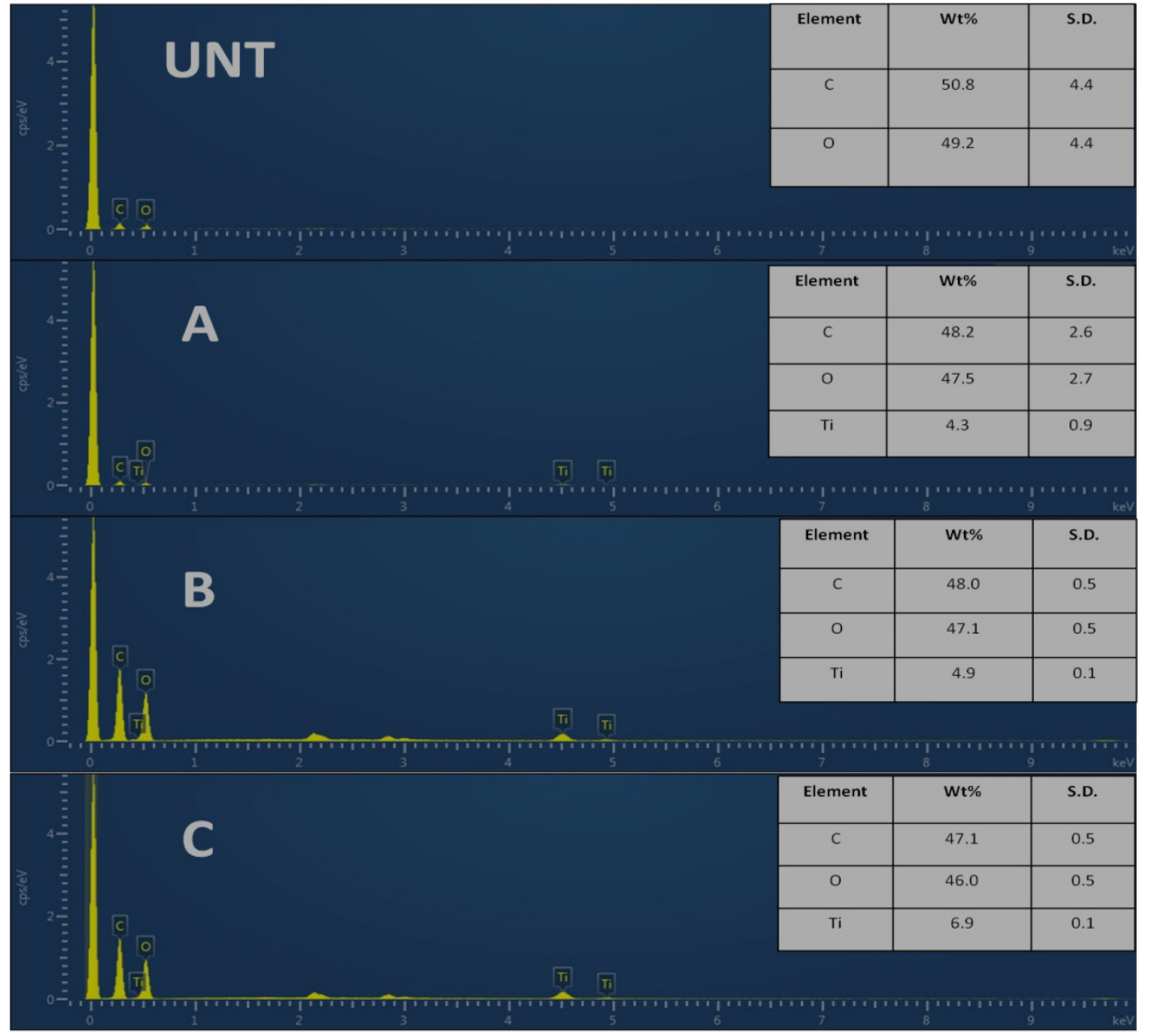

Figure 2. EDX spectra of $\mathrm{TiO}_{2}$ coated cotton fabrics prepared by various methods (UNT) Untreated, (A) PDC, (B) PDH and (C) PDS

It can be observed that the values of Ti content measured through EDX technique (referring to Fig. 2) are much higher than the values obtained from ICP-MS method for the same set of samples. This anomaly can be attributed to the surface deposition behavior of $\mathrm{TiO}_{2}$ by these routes. In case of 
ICP method, the values of Ti content is measured with respect to the cotton fabric weight by completely disintegrating the substrate into acid. In contrast, in case of EDX, it is calculated taking into consideration only the surface layer deposition.

\subsection{UV protection properties of treated fabrics}

The UV protection property of the fabric is quantitatively expressed in terms of UV protection factor (UPF) values and a UV protection rating is given to the fabric in accordance to the AATCC 183:2010 standards. UPF rating above 50 is considered as excellent. The UPF value of the untreated cotton fabric was found to be 8.9. In comparison to the untreated fabric, $\mathrm{TiO}_{2}$ treated fabric always had a higher UPF values. However, the value of UPF is largely dependent upon the treatment procedure.

The UPF of the $\mathrm{TiO}_{2}$ treated samples prepared by PDC, PDH and PDS are given in Table 2 . The curing time for PDC in sample A was fixed as $3 \mathrm{~min}$. The treatment time given in PDH and PDS for sample $\mathrm{B}$ and $\mathrm{C}$ was $3 \mathrm{~h}$.

Table 2. The UV protection parameters of cotton fabrics prepared by different methods (after $1^{\text {st }}$ standard wash)

\begin{tabular}{|c|c|c|c|c|c|}
\hline Sample code & Process & UPF mean & T\% (UVA) & T\% (UVB) & UPF rating \\
\hline UNT & -- & $8.9(6.9 \%)$ & $17.5(5.9 \%)$ & $9.9(7.3 \%)$ & 10 \\
\hline A & PDC & $50.5(5.8 \%)$ & $10.8(6.0 \%)$ & $1.0(7.8 \%)$ & 50 \\
\hline B & PDH & $69.1(6.2 \%)$ & $7.1(5.6 \%)$ & $0.9(5.4 \%)$ & 65 \\
\hline C & PDS & $114.2(7.9 \%)$ & $6.8(6.5 \%)$ & $0.3(7.6 \%)$ & 105 \\
\hline
\end{tabular}

As can be seen from the Table 2, the UPF values of sample A are lower than that of sample B and C. Hence for better UV protection, the PDH/PDS route should be chosen over the PDC route.

Comparing the add-on levels of the three samples, it can be easily seen that the add-on levels of $A$ and $B$ are similar, whereas the level of deposition in sample $C$ is much higher, which can be correlated to its higher UPF value. The reason for the high add-on value in sample $C$ lies in the fact that the thermal treatment to the fabric sample is being given in a medium of $\mathrm{TiO}_{2}$ precursor. Even if a small fraction of this gets deposited on fabric, it can significantly enhance the add-on levels on fabric, leading to higher UPF values.

The UPF mean of fabric samples were measured after $1^{\text {st }}$ and $5^{\text {th }}$ standard wash. As shown in Fig. 3, the UPF value goes down from 50.5 to 40.3 after 5 consecutive washes in sample A (almost $20 \%$ decrease). This decrease in UPF is about $14 \%$ in sample C (the UPF mean value goes down from 114.2 to 98.8 after 5 standard washes). Among all, sample B shows the best result in terms of wash durability and \% decrease in UPF mean value. The \% decrease in UPF mean was recorded about $7 \%$ after 5 standard washes (the UPF mean value decreased from 69.1 to 65.2 after 5 standard washes). 


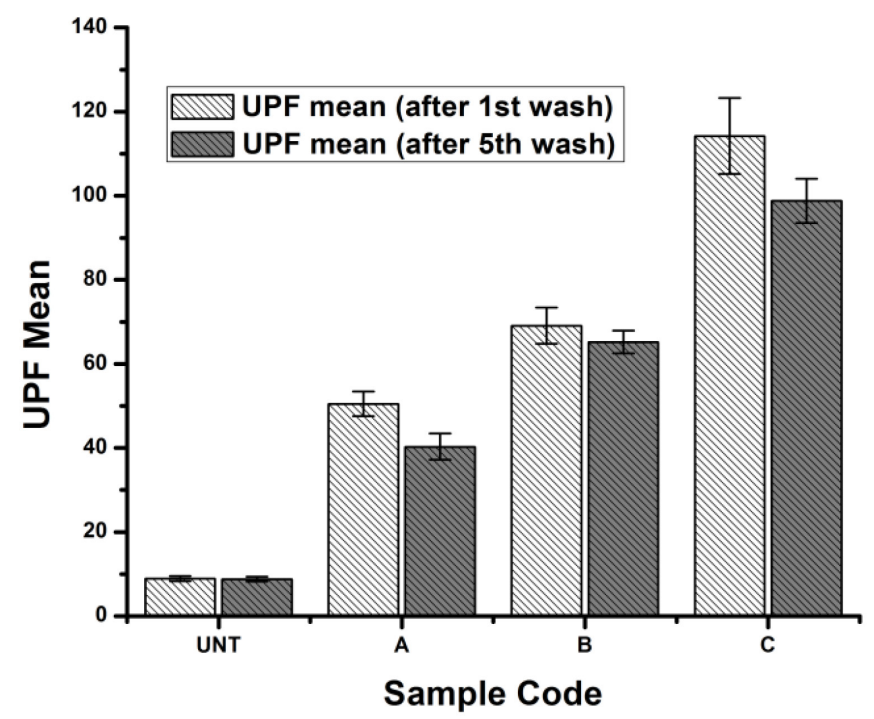

Figure 3. UPF mean of fabric samples after $1^{\text {st }}$ and $5^{\text {th }}$ standard wash

\section{Conclusions}

The present study compares the deposition behavior of $\mathrm{TiO}_{2}$ on cotton fabric by adopting three different process routes, i.e., pad-dry, pad-dry-hydrothermal and pad-dry-solvothermal. $\mathrm{The}^{\mathrm{TiO}_{2}}$ coating was done on cotton fabric by using its pre-cursor through modified sol-gel method. The morphological structures of the coatings confirmed that the process routes play a critical role in deposition behavior of $\mathrm{TiO}_{2}$ on textile surface. The extent of titania deposition also depends upon the process route followed, which in turn determines the level of UV protection factor in fabric. The results showed that a very thin wash durable coating of $\mathrm{TiO}_{2}$ on cotton is possible by adopting either hydrothermal or solvothermal route. Even a very low add-on level of $\mathrm{TiO}_{2}$ is effective in providing good UV protection to the treated fabrics.

\section{References}

[1] C.-H. Xue et al., Superhydrophobic conductive textiles with antibacterial property by coating fibers with silver nanoparticles, Applied Surface Science. 258(7) (2012) 2468-2472.

[2] Y. Gao, R. Cranston, Recent advances in antimicrobial treatments of textiles, Textile Research Journal. 78(1) (2008) 60-72.

[3] J. Alongi, F. Carosio, G. Malucelli, Current emerging techniques to impart flame retardancy to fabrics: an overview, Polymer Degradation and Stability. 106 (2014) 138-149.

[4] Y.-L. Zhang et al., Recent developments in superhydrophobic surfaces with unique structural and functional propertyes, Soft Matter. 8(44) (2012) 11217-11231.

[5] M.L. Gulrajani, G. Deepti, Emerging techniques for functional finishing of textiles, Indian Journal of Fibre and Textile Research. 36 (2011) 388-397.

[6] M.H. Zohdy et al., Novel UV-protective formulations for cotton, PET fabrics and their blend utilizing irradiation technique, European Polymer Journal. 45(10) (2009) 2926-2934.

[7] G. Reinert et al., UV-protecting properties of textile fabrics and their improvement, Textile Chemist \& Colorist. 29(12) (1997).

[8] O.K. Alebeid, T. Zhao, Review on: developing UV protection for cotton fabric, The Journal of The Textile Institute. (2017) 1-13. 
[9] Ž. Senić et al., Application of $\mathrm{TiO}_{2}$ nanoparticles for obtaining self-decontaminating smart textiles, Scientific Technical Review. 61(3-4) (2011) 63-72.

[10] S. Gowri et al., Polymer nanocomposites for multifunctional finishing of textiles-a review, Textile Research Journal. 80(13) (2010) 1290-1306.

[11] A. Mishra, B.S. Butola, Development of cotton fabrics with durable UV protective and selfcleaning property by deposition of low $\mathrm{TiO}_{2}$ levels through sol-gel process, Photochemistry and Photobiology. 94(3) (2018) 503-511.

[12] L. Karimi et al., Effect of $\mathrm{Nano}^{\mathrm{TiO}_{2}}$ on self-cleaning property of cross-linking cotton fabric with succinic acid under UV irradiation, Photochemistry and Photobiology. 86(5) (2010) 10301037.

[13] C.-C. Chen, C.-C. Wang, Crosslinking of cotton cellulose with succinic acid in the presence of titanium dioxide nano-catalyst under UV irradiation, Journal of Sol-Gel Science and Technology. 40(1) (2006) 31-38.

[14] K. Sundaresan et al., Influence of nano titanium dioxide finish, prepared by sol-gel technique, on the ultraviolet protection, antimicrobial, and self-cleaning characteristics of cotton fabrics, Journal of Industrial Textiles. 41(3) (2012) 259-277.

[15] S.S. Uur, M. Sariisik, A. Hakan Aktas, Nano-TiO2 based multilayer film deposition on cotton fabrics for UV-protection, Fibers and Polymers. 12(2) (2011) 190.

[16] B. Pranaityte, A. Padarauskas, E. Naujalis, Application of ICP-MS for the determination of trace metals in textiles, Chemija. 18(3) (2007). 